\title{
EFFECTS OF PRE-BUCKLING DEFLECTIONS ON LOCAL, DISTORTIONAL AND LATERAL-TORSIONAL BUCKLING OF I -BEAMS
}

\author{
By Seizou USUKI* and Kaoru HASEBE**
}

\begin{abstract}
The displacement and strain distributions for a plate segment comprising open cross section are derived considering the pre-buckling deflection of beam axis. Applying the higher order theory obtained to I -beams bent about their major axes by equal end moments, the effects of pre-buckling deflections on local, distortional and lateral-torsional buckling are investigated. Solving eigen-value problems, it is clarified that the stress corresponding to distortional buckle of an I -beam is conservative compared with that neglecting the pre-buckling deflections and the stress corresponding to lateral-torsional buckle occuring at long lengths are progressive compared with the results of Timoshenko.
\end{abstract}

\section{INTRODUCTION}

The coupling analyses of local, distortional and lateral-torsional buckling of thin-walled beams have been developed by finite element or finite strip metod as well as theoretical methods. It will be however difficult to consider the effects of major axis deflections of, for instance, I -beams subjected to bending moments, by the above numerical methods as well as theoretrical ones.

The writers have presented a new theory ${ }^{1)}$, which can treat the coupling of local, distortional and lateral-torsional buckling and can consider the effects of pre-buckling deflections. The previous paper by the writers is unfortunately based on a second order theory and the second order terms of pre-buckling deflections and of initial stresses acting on the cross sections of I -beams are neglected in the incremental variational formulation.

In this paper, general non-linear displacement and strain destributions for a plate segment comprising open cross section are derived without neglecting higher order terms of pre-buckling deflections. Applying the higher order theory obtained to I -beams bent about major axes, the effects of pre-buckling deflections on buckling stresses or moments are investigated.

\section{INCREMENTAL FORM FOR EACH PLATE SEGMENT}

\section{(1) Web plate}

From the assumption of no distortion of cross section before buckling and considering $h_{1}^{0}(s, z)$ defined by Eq. (28) in Ref. 1) equals to zero, it holds true

* Member of JSCE, Dr. Eng., Prof., Dept. of Civil Eng., Akita Univ.

** Member of JSCE, Ms. Eng., Assist. Prof., Dept. of Civil Eng., Akita Univ. (Tegata-Gakuen-cho, 1-1, Akita-shi, 010, Japan. ) 


$$
\eta^{* 0}=\eta_{1}^{0}=0, \quad \xi^{* 0}=\xi_{1}^{0}=\xi_{0}^{0}(z)
$$

where $\xi_{0}^{0}$ is the value of the tangential displacement $\xi^{* 0}$ at the middle point of the web (Fig. 1); in other words $\xi_{0}^{0}$ is the pre-buckling deflection of beam axis, $z$. Then, the variation of axial displacement after buckling, $\delta\left(w_{1}^{0}+w_{1}\right)$ at the nodal line, $i=1$ are represented by the displacements at the middle point of the web plate as

$$
\begin{aligned}
\delta\left(w_{1}^{0}+w_{1}\right)= & \delta\left(w_{0}-l_{1} \xi_{0}^{\prime} / 2\right) \\
& \left.+\delta \underline{\underline{\xi_{0}^{0 \prime} h_{10}}}-l_{1} h_{10}^{\prime} / 2+f_{10}\right) \cdots
\end{aligned}
$$
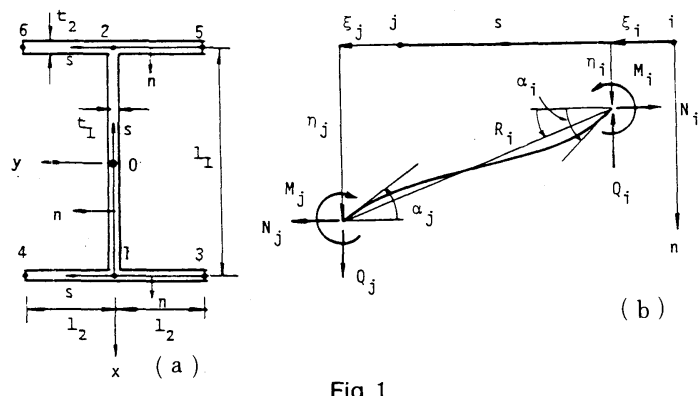

( b )

Fig. 1

where

$$
\left[\begin{array}{ll}
h_{10} & h_{12} \\
f_{10} & f_{12}
\end{array}\right]=\left[\begin{array}{ll}
h_{1}\left(l_{1} / 2, z\right), & h_{1}\left(l_{1}, z\right) \\
f_{1}\left(l_{1} / 2, z\right), & f_{1}\left(l_{1}, z\right)
\end{array}\right]
$$

and the function $f_{1}$ and $h_{1}$ are the second order in incremental quantities and the same as Eq. (28) in Ref. 1).

In the right hand side of Eq. (2), for simplicity, the superscript ( $)^{*}$ is abrebiated. In Eq. (2), the terms on the double under line were neglected in the previous paper.

The variation of strain, $\delta\left(\varepsilon_{z}^{0}+\varepsilon_{z}\right)$ is also obtained as

$$
\delta\left(\varepsilon_{z}^{0}+\varepsilon_{z}\right)=\delta \varepsilon_{z}^{L}+\delta \varepsilon_{z}^{N}
$$

where

$$
\begin{aligned}
\varepsilon_{z}^{L}= & w_{0}^{\prime}+\xi_{0}^{0} \xi_{0}^{\prime}+\left(l_{1} / 2-s\right) \xi_{0}^{\prime \prime}+n\left(\xi_{0}^{0 \prime \prime} \alpha-\eta^{* \prime \prime}\right) \\
\varepsilon_{z}^{N}= & -\left(l_{1} / 2-s\right) h_{10}^{\prime \prime}+n \xi_{0}^{\prime \prime} \alpha+\left(\xi_{0}^{\prime 2}+\eta^{* \prime 2}+n^{2} \alpha^{\prime 2}\right) / 2+f_{10}^{\prime}-f_{1}^{\prime}(s, z) \\
& +\underline{\xi}_{0}^{0 \prime}\left(h_{10}-h_{1}(s, z)\right)
\end{aligned}
$$

The terms on the single under line govern the behavior of web plate in the $s^{-} z$ plane and do not concern with the buckle. The terms on the double under line were neglected in the previous paper.

\section{(2) Flange plates}

For the flange plates, the conventional assumption that the flanges remain undistorted in the post-buckling state is employed. For the pre-buckling state, the displacements in the $n$ and $s$ directions (see Fig. 1) are given as

$$
\xi^{* 0}=0, \quad \eta^{* 0}=-\xi_{0}^{0}(z)
$$

From continuity conditions of displacements at nodal line 1,2 , and 0 the tangential and normal displacements after buckling are given by displacements of point 0 .

The variations of strain, $\delta\left(\varepsilon_{z}^{0}+\varepsilon_{z}\right)$ after buckling are obtained as

$$
\delta\left(\varepsilon_{z}^{0}+\varepsilon_{z}\right)=\delta \varepsilon_{z}^{L}+\delta \varepsilon_{z}^{N}
$$

where for the lower flange, $\varepsilon_{z}^{L}$ and $\varepsilon_{z}^{N}$ are given as

$$
\begin{aligned}
& \varepsilon_{z}^{L}=\underline{w_{0}^{\prime}+\xi_{0}^{0 \prime} \xi_{0}+\left(l_{1} / 2-n\right) \xi_{0}^{\prime \prime}}+\left(l_{2}-s\right)\left(\eta_{1}^{\prime \prime}+n \alpha_{1}^{\prime \prime}-\underline{\underline{\xi_{0}^{0 \prime \prime} \alpha_{1}}}\right. \\
& \varepsilon_{z}^{N}=-\left(l_{1} / 2+n\right) h_{10}^{\prime \prime}+f_{10}^{\prime}-\left(l_{2}-s\right) \xi_{0}^{\prime \prime} \alpha_{1}+n\left(\eta_{1}^{\prime \prime} \alpha_{1}-\underline{\underline{\xi_{0}^{0 \prime \prime} \alpha_{1}^{2}}} / 2\right)+\left[\eta_{1}^{\prime 2}+\xi_{0}^{\prime 2}\right. \\
& \left.+\left\{\left(l_{2}-s\right)^{2}+n^{2}\right\} \alpha_{1}^{\prime 2}\right] / 2+\underline{\underline{\xi_{0}^{0 \prime \prime} h_{10}}}
\end{aligned}
$$

and for the upper flange

$$
\begin{aligned}
\varepsilon_{z}^{L}= & w_{0}^{\prime}+\xi_{0}^{0} \xi_{0}-\left(l_{2} / 2-n\right) \xi_{0}^{\prime \prime}+\left(l_{2}-s\right)\left(\eta_{1}^{\prime \prime}+l_{1} R_{1}^{\prime \prime}+n \alpha_{2}^{\prime \prime}-\underline{\underline{\left.\xi_{0}^{0 \prime} \alpha_{2}\right)}}\right. \\
\varepsilon_{z}^{N}= & \left(l_{1} / 2-n\right) h_{10}^{\prime \prime}+n h_{12}^{\prime \prime}+f_{10}^{\prime}-f_{12}^{\prime}-\left(l_{2}-s\right) \xi_{0}^{\prime \prime} \alpha_{2}+n\left\{\left(\eta_{1}^{\prime \prime}+l_{1} R_{1}^{\prime \prime}\right) \alpha_{2}-\underline{\underline{\left.\xi_{0}^{0 \prime} \alpha_{2}^{2} / 2\right\}}}\right. \\
& +\left[\left(\eta_{1}^{\prime}+l_{1} R_{1}^{\prime}\right)^{2}+\xi_{0}^{\prime 2}+\left\{\left(l_{2}-s\right)^{2}+n^{2}\right\} \alpha_{2}^{\prime 2}\right] / 2+\underline{\underline{\xi_{0}^{0 \prime}\left(h_{10}-h_{12}\right)}}
\end{aligned}
$$

The terms on the single under-line do not consern with buckling behaviors. The terms on the double under-lines were neglected in the previous paper.

\section{BUCKLING EQUATIONS}

The differential equations governing buckling behaviors of an I-beam subjected to equal end moments can 
be derived by the use of an incremental variational principle. The initial normal stress, $\sigma_{z}^{0}$ acting on the cross section and the pre-buckling deflection $\xi_{0}^{0}(z)$ of beam axis are obtained from the elementary beam theory.

The unknown nodal parameters of the problem are $\boldsymbol{\alpha}^{T}=\left[\begin{array}{llll}\alpha_{1} & \alpha_{2} & \eta_{0} / l_{1} & R_{1}\end{array}\right]$ and this is the same as the previous paper. If the $I$-beam is simply supported, the solution of the fourth order differential equation governing the parameter $\alpha$ becomes $\alpha=\boldsymbol{u} \cdot \sin n \pi z / L$, where $\boldsymbol{u}$ is a buckling mode and $L$ is a span length. From the condition of non-zero values of $\boldsymbol{u}$, the eigen-value problem is obtained as

$$
\left|\boldsymbol{K}+k \boldsymbol{M}+k^{2} \boldsymbol{N}\right|=0
$$

where $\boldsymbol{K}$ and $\boldsymbol{M}$ are the same as the previous paper (Eqs. (76) and (77) in Ref. 1)) and $k$ is the buckling coefficient defined by $k=\sigma_{c r} l_{1}^{2} t_{1} /\left(\pi^{2} E t_{1}^{3} / 12\right)$. The matrix $N$ is newly introduced and is given by

$$
\begin{aligned}
N= & \left(\frac{2 \alpha \gamma \beta^{2}}{3 n \delta}\right)^{2}\left[\begin{array}{cccc}
2 / 15+\delta, & -1 / 30, & 0, & -1 / 10 \\
& 2 / 15+\delta, & 0, & -1 / 10 \\
\text { SYM. } & 0, & 0 \\
& 6 / 5
\end{array}\right] \\
& +\left(\frac{\alpha}{6 n}\right)^{2} \frac{12 \gamma \beta^{2}}{\delta}\left[\begin{array}{cccc}
1 / 35+4 \gamma / 15, & -1 / 210-\gamma / 15, & 0, & -2 / 35-\gamma / 5 \\
& 1 / 35+4 \gamma / 15, & 0, & -2 / 35-\gamma / 5 \\
\text { SYM. } & & 0, & 0 \\
& & 18 / 35+12 \gamma / 5
\end{array}\right]
\end{aligned}
$$

where $\alpha=L / l_{1}$ and $n$ is the half wave number. The parameters, $\gamma, \beta$ and $\delta$ are non-dimensional parameters on the cross sectional dimesions of I -beams and are the same as the previous paper. The smallest eigen-value of Eq. (10) defines the critical stress, $k_{c r}$ and the corresponding eigen-vector defines the buckled shape.

\section{RESULT AND DISCUSSION}

Fig. 2 is produced by calculating buckling end moments. The buckling moments are not mimimum values, but the values for a half wave length $(n=1)$. The geometry of I-beam is shown in Fig. 2. At the peak points $\mathrm{C}$ of the curves where the distortional buckling occurs, the critical end moment by the present theory is conservative by $17 \%$ than that neglecting the pre-buckling deflections. For a comparison, the lateral-torsional buckling curves by Trahair and Woolcock ${ }^{2)}$, by Nishino et al. ${ }^{3)}$ and by Timoshenko and Gere ${ }^{4)}$ are also illustrated in Fig. 2.

Fig. 3 shows the case of cross section 2 which has doble flange width of the cross section 1 . In the range of beam length, $L / h>20$, the present results are agree with those by Trahair and Nishino. The values of critical end moments by neglecting the pre-buckling deflections are conservative by $12 \%$ at $L / h=20$ in comparison with the present results, and Trahair and Nishino. This is due to the fact that the ratio of bending rigidity, $E I_{x} / E I_{y}$ affecting extremely the critical moments has the large value of $22 \%$ for the cross section 2 (the cross section 1 in Fig. 2 has the value of $5.0 \%$ ).

Fig. 4 shows the critical stresses $\sigma_{c r} / E$ for various half wave numbers. The Gothic line shows minimum crirical stresses for given beam lengths, $L / h$ and the dotted line shows the results neglecting the pre-buckling deflections for $n=1$ (these are also agree with finite strip method).

Fig. 5 demonstrates the critical stresses $\sigma_{c r} / E$ versus $t_{f} / b$ or $E I_{x} / E I_{y}$, for the typical beam length, $L / h=15$. The ratios, $h / t_{w}$ and $t_{f} / t_{w}$ are fixed to 34.0 and 1.46 , respectively and these values are the same as the cross section, 1 and 2 .

In the case of Trahair and Nishino, the critical stresses become infinitely large at $E I_{x} / E I_{y}=1:$ in other words the lateral-torsional buckle can not occurs, in the range $E I_{x} / E I_{y}>1$. Whereas, as can be seen, the present theory can predict the local buckling behavior in the range $E I_{x} / E I_{y}>1$. The results by Timoshenko and Gere shows the lateral-torsional buckle occurs, no matter how $E I_{x} / E I_{y}$ become large. 


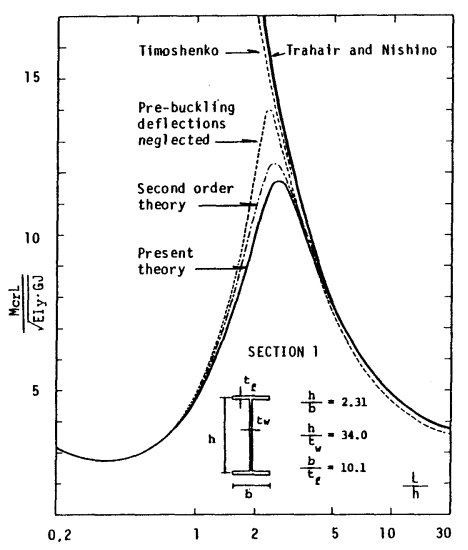

Fig. 2 Effects of pre-buckling deflections on buckling moments for section 1.

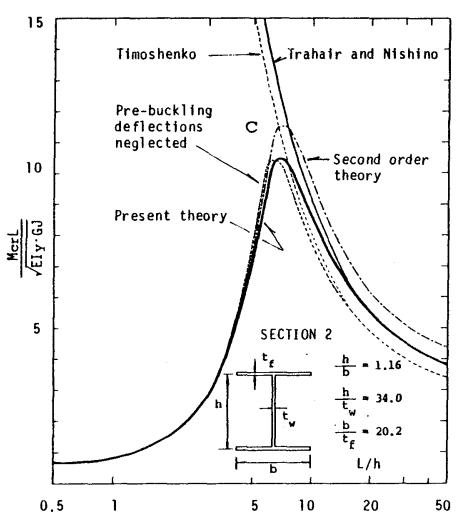

Fig. 3 Effects of pre-buckling deflections on buckling moments for section 2 .

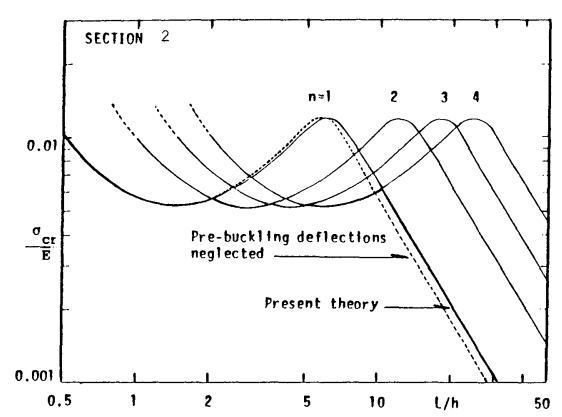

Fig. 4 Critical stress for half wave numbers, $n$ and minimum critical stress

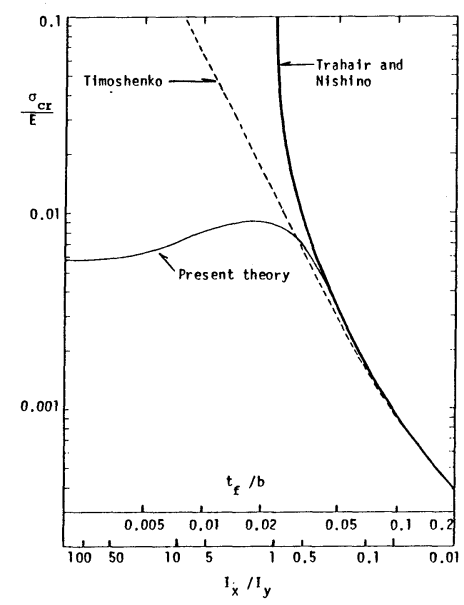

Fig. 5 Effects of the ratio $I_{x} / I_{y}$ on buckling stress.

\section{CONCLUSIONS}

A higher order theory governing the elastic local, distortional and lateral-torsional buckling of I-beams subjected to bending moments are presented, considering all of terms on the pre-buckling deflections. In this paper, I -beams with web width-thickness ratio of 34 are examined.

The distrtional critical moment obtained by the present theory for sections with low flange width-thickness ratio (section 1) is $17 \%$ lower than that by neglecting pre-buckling deflections. The values of minimum critical moments for various beam lengths are however affected only in the range of relatively long beams. When the ratios of flexural figidity about weak axis to that about major axis, $E I_{x} / E I_{y}$ become larger than one, the distortional and local buckle occur and the lateral-torsional buckle can not be observed.

\section{REFERENCES}

1) Usuki, S. and Hasebe, K. : Local and distortional buckling of thin walled beams based on second order displacement fields, ProC. JSCE, No. 344/ I-1, pp. 357 366, 1984.

2) Trahair, N. S. and Woolcock, S. T. : Effects of major axis curvature on I -beam stability, Proc. ASCE, Vol. 99, No. EM 1, pp. 85 98, 1973 .

3) Nisino, F., Kurakata, Y., Hasegawa, A. and Okumura, T. : Thin-walled members under axial force, bending and torsion Proc. JSCE, No. 247, pp.9〜19, 1976.

4) Timoshenko, S. P. and Gere, J.M. : Theory of elastic stability, McGraw-Hill, N. Y., 1959. 